\title{
\begin{tabular}{l|l} 
POLITIQUES \& & Politiques et management public
\end{tabular}

\section{L'information médico-économique entre décision stratégique et action publique : tout savoir pour peu pouvoir!}

Clinical-Economic information and the Gap between Strategic decision making and Public action : so little yield for knowing so much

Christophe Pascal, Jean-Baptiste Capgras, Nicolas Guilhot et JeanPierre Claveranne

\section{OpenEdition}

\section{Journals}

Édition électronique

URL : http://journals.openedition.org/pmp/4167

ISSN : 2119-4831

Éditeur

Institut de Management Public (IDPM)

\section{Édition imprimée}

Date de publication : 15 mars 2011

ISSN : 0758-1726

Référence électronique

Christophe Pascal, Jean-Baptiste Capgras, Nicolas Guilhot et Jean-Pierre Claveranne, «L'information médico-économique entre décision stratégique et action publique : tout savoir pour peu pouvoir ! », Politiques et management public [En ligne], Vol 28/1 | 2011, mis en ligne le 05 octobre 2012, consulté le 20 avril 2019. URL : http://journals.openedition.org/pmp/4167 


\title{
L'information médico-économique entre décision stratégique et action publique : tout savoir pour peu pouvoir!
}

\author{
Christophe Pascal *, Jean-Baptiste Capgras, \\ Nicolas Guilhot, Jean-Pierre Claveranne \\ Enseignants-Chercheurs au GRAPHOS, \\ IFROSS - Université Jean Moulin Lyon, 318 rue Chevreul, 69007 Lyon
}

\section{Résumé}

À partir d'une série de recherches-interventions menées depuis 1990, cet article critique les apports de la transparence de l'activité des hôpitaux publics sur leur pilotage. Paradoxalement, alors que les acteurs possèdent une connaissance quasi-exhaustive de la production hospitalière avec le Programme de médicalisation des systèmes d'information (PMSI), les autorités de régulation et les hôpitaux publics font face à la difficulté de mettre en œuvre les décisions découlant des usages sophistiqués de cette connaissance. En effet, la politique de régulation de l'offre sur un territoire s'oppose aux incitations concurrentielles de la Tarification à l'activité, tandis que la stratégie concurrentielle des établissements publics est limitée par leurs problèmes de recrutement de la ressource médicale et par la rigidité de leurs coûts de production. Les cliniques privées, du fait de leur plasticité, apparaissent en retour comme les principales bénéficiaires de ce système d'information.

(c) 2011 IDMP/Lavoisier SAS. Tous droits réservés

Mots clés : information, hôpital, PMSI, régulation, stratégie, concurrence.

\section{Abstract}

Clinical-Economic Information and the Gap Between Strategic Decision Making and Public Action: So Little Yield for Knowing So Much. Based on action research accumulated since 1990, this paper analyzes the limited impact of the French medical information system [Program of medicalization of information system (PMSI)] on the strategic management capability of the public hospital sector. Whereas the PMSI provides nearly exhaustive knowledge of hospital activities, regulatory authorities and public hos-

\footnotetext{
*Auteur correspondant : christophe.pascal@univ-lyon3.fr doi:10.3166/pmp.28.57-82 @ 2011 IDMP/Lavoisier SAS. Tous droits réservés
} 
pital administrators are unable to act on this knowledge that is often highly sophisticated. Supply side regulation of regional markets contrasts with the competitive pressure arising from the prospective payment system. The competitive strategy of the public hospital is limited by the difficulties of recruiting medical resources and by the rigidity of their production costs. On the other hand, thanks to their malleability, private hospitals appear to be the main beneficiaries of this information system. @ 2011 IDMP/Lavoisier SAS. Tous droits réservés

Keywords : Information; hospital; DRG; case-mix reimbursement; regulation; strategy; competition.

Depuis son origine, l'hôpital a toujours éprouvé de grandes difficultés pour trouver les ressources nécessaires à son fonctionnement, mais depuis le déclin de la conception charitable des soins au $19^{\mathrm{e}}$ siècle, ses difficultés portent également sur la justification de ses besoins financiers (Guilhot N., 2008). Ouvrir la « boîte noire » de l'hôpital est alors devenu l'objectif des financeurs, qu'ils relèvent de la bienfaisance ou de l'État. Sous la pression de la nouvelle tutelle préfectorale imposée aux hôpitaux sous le Consulat, la mise en place d'outils de gestion administrative aboutit lentement à l'apparition d'un début d'économat dont l'objet était essentiellement de chiffrer des prix de journée prévisionnels. Une des dynamiques de l'histoire de l'hôpital a été la recherche de la maîtrise de l'information permettant de dépasser la simple administration pour envisager la gestion. Beaucoup plus récemment, dans les années 1980, un système de description de l'activité s'est constitué à partir du Programme de médicalisation des systèmes d'information (PMSI). Ce système est devenu tellement performant que l'on assiste aujourd'hui à une véritable contradiction. Chaque acteur du secteur est en effet en mesure de tout savoir sur la production hospitalière sans pour autant avoir les capacités d'agir sur tous les déterminants de cette dernière : « tout savoir pour peu pouvoir» tel est ainsi le paradoxe de l'information médico-économique.

La question qui se pose alors est d'examiner les apports de la transparence de l'activité des hôpitaux publics instaurée par le PMSI sur leur pilotage. L'objectif de cet article est de montrer en quoi ce système d'information, en dépit de la pertinence des connaissances qu'il fournit pour les autorités administratives comme pour les établissements, est finalement de peu d'utilité pour la définition des politiques de régulation et des stratégies des établissements publics. Après avoir dépeint l'émergence de l'information sur la production hospitalière (1), les fonctionnalités actuelles et potentielles du PMSI pour la description et l'analyse de l'offre de soins sont présentées (2). Enfin, les obstacles et les freins à une exploitation effective de cette information pour la décision publique sont discutés (3). 


\section{Encart méthodologique}

Cet article repose sur l'expérience collective accumulée au cours d'une série de recherches-interventions conduites entre 1990 et 2009. En particulier, l'élaboration des Schéma régionaux d'organisation sanitaire (SROS) de deuxième et troisième générations a été conduite pour le compte de deux Agences régionales de l'hospitalisation (ARH) différentes ainsi que l'accompagnement de l'une d'entre elles dans la définition des objectifs quantifiés de l'offre de soins (OQOS). Quatre recherches ont été menées pour le compte d'une Direction départementale de l'action sanitaire et sociale (DDASS) et de deux ARH afin de les assister dans l'organisation territoriale de l'offre de soins hospitalière en 2005, 2006 et 2008. Une étude sur les opérations de recomposition hospitalière a également été conduite pour le compte du ministère de la santé entre 2005 et 2007. En 1994, notre équipe a participé pour le compte de l'Assurance-maladie à l'expérimentation « Languedoc-Roussillon » sur le financement des établissements de santé à partir du PMSI. Enfin, une dizaine d'établissements, publics et privés, nous ont missionné pour les assister dans l'élaboration de leurs projets d'établissement et/ou la réalisation d'un diagnostic stratégique notamment en vue de fusions d'établissements et de regroupements d'activités.

En plus de ces recherches-interventions conduites en prise directe avec les acteurs et les problématiques de terrain, cet article s'appuie sur le projet de recherche $\mathrm{BIOTOP}^{2} \mathrm{ES}^{1}$ lancé en 2008 et dont la première phase s'est achevée en 2010. Cette première phase a notamment constitué le socle d'une collaboration avec l'éditeur du logiciel PMSI Pilot, leader français des logiciels de traitement du PMSI dans le secteur hospitalier public (plus de 350 établissements de santé publics équipés).

\section{L'information sur l'hôpital : une lente construction}

L'offre de soins hospitaliers française, qu'elle soit publique ou privée, résulte de l'interaction de quatre dimensions : la dimension historique, la dimension technologique, la dimension régulatrice, et la dimension stratégique.

L'histoire pluri-centenaire des hôpitaux a inscrit siècle après siècle les établissements dans des lieux et des territoires bien déterminés, ancrés dans les habitudes des populations et intégrés dans les schémas de réflexion et de décision de l'Administration en tant qu'éléments structurant de l'espace public. Les évolutions différenciées de ces espaces, en termes de démographie, de modes de vie, de déplacements domicile-travail, et de développement économique, conjuguées aux contraintes architecturales inhérentes aux transformations du soin et à l'intégration de plateaux techniques de plus en plus étendus, ainsi qu'aux aspirations à l'autonomie et à l'indépendance des acteurs hospitaliers et des élus, ont conduit à la constitution peu maîtrisée d'un maillage dense d'établissements sanitaires publics.

Dans les quarante dernières années, l'explosion technologique, couplée à l'évolution de la pratique médicale, a bouleversé l'ordre pluri-centenaire. La pratique médicale que

\footnotetext{
${ }^{1}$ Benchmark inter-organisationnel territorial de l'offre publique et privée des établissements de santé.
} 
permettent les nouvelles technologies conduit à un accroissement des disciplines médicales autour d'un plateau technique de plus en plus sophistiqué et coûteux, le colloque pluriel prend le pas sur le colloque singulier, l'architecture pavillonnaire s'efface derrière des structures architecturales compactes. Se dessine au cours des années une coupure de plus en plus forte entre une médecine toujours en évolution et une organisation d'un autre temps, où l'administration prime sur la gestion malgré les marges de manœuvre dont jouissent au moins dans les textes, les directions hospitalières, et malgré le fameux amendement liberté promulgué lors de la loi de 1991 laissant aux directeurs d'hôpitaux la possibilité d'adapter l'organisation de l'hôpital aux contingences locales.

L'offre de soins qui en résulte s'avère peu satisfaisante en termes d'accessibilité, de qualité et de coût : la continuité des prises en charge est mise à mal par l'éclatement des structures et des acteurs, tandis que la sous-utilisation des plateaux techniques, la redondance des actes et des activités, et la non-coordination augmentent le coût global du système. Conscient de ces difficultés, l'État s'est engagé dès le début des années soixante dans une politique de régulation de l'offre visant à promouvoir une meilleure répartition des équipements sur le territoire national au travers de la planification sanitaire.

Pour remédier à ces mouvements structurels forts, les autorisations administratives d'installation constituent la première pierre de l'édifice régulation : une autorisation est ainsi imposée pour la création des hôpitaux publics par une ordonnance de 1958, de même qu'une déclaration préalable pour la création ou l'extension des cliniques privées. Ce régime a été progressivement renforcé et étendu à l'ensemble des titulaires d'équipements « lourds » et des cliniques privées, avant d'être réformé et simplifié en septembre 2003 au profit d'autorisations d'activités de soins (18 activités possibles) et d'équipements lourds (5 types d'équipements).

La carte sanitaire créée par la loi hospitalière du 31 décembre 1970 découpe l'espace géographique en 256 secteurs sanitaires, répartis en 21 régions. Elle vise ainsi à mettre en adéquation les équipements, appréciés en lits et places pour 100000 habitants, avec les besoins de la population de chaque secteur. Le Schéma régional d'organisation sanitaire (SROS), instauré par la loi hospitalière du 31 juillet 1991, vient ajouter un volet plus qualitatif et prospectif au dispositif, qui vise à une répartition optimale des installations et des structures de santé déterminées par la carte sanitaire. Suite à l'ordonnance du 4 septembre 2003, ce schéma est devenu l'instrument unique de planification, qui consacre désormais le territoire de santé en lieu et place du secteur sanitaire. Fondé sur une évaluation des besoins de santé physique et mentale de la population, le SROS définit une offre-cible de soins qui trouve sa traduction notamment sous la forme d'Objectifs quantifiés de l'offre de soins (OQOS) exprimés sous forme de nombre d'implantations ou de volume de soins autorisés au sein de chaque zone du territoire de santé. Ces objectifs quantifiés sont ensuite déclinés pour chaque titulaire d'autorisation d'activité de soins et d'équipements lourds dans le cadre des contrats pluriannuels d'objectifs et de moyens instaurés par l'ordonnance portant réforme de l'hospitalisation publique et privée d'avril 1996, que ces derniers signent avec l'Agence régionale de l'hospitalisation (ARH), puis l'Agence régionale de santé (ARS) depuis avril 2010, pour une durée maximale de 5 ans.

Parallèlement à ce mouvement de planification, s'est élaboré un ensemble de mesures législatives visant à développer l'autonomie et la responsabilisation des établissements : rédaction obligatoire d'un projet d'établissement (1991), contrats quinquennaux pluriannuels d'objectifs et de moyens passés avec les agences régionales (1996), structuration interne en pôles d'activités médicaux et contractualisation interne de ces pôles avec la direction (2007). 
La réforme du système de financement des établissements participe de ce mouvement de responsabilisation : la dotation globale de financement attribuée aux hôpitaux publics depuis 1983 (Hatchuel A., Moisdon J.-C. et Molet H., 1988) cède la place à partir de 2004 à la tarification à l'activité (T2A) (Claveranne J.-P. et Pascal C., 2005). Appliqué uniformément aux hôpitaux et aux cliniques, mais avec deux échelles tarifaires distinctes, le nouveau système constitue une révolution dans la gestion des établissements publics, en posant comme référentiel central de la performance hospitalière la recherche de l'efficience. Ce sont désormais les ressources obtenues grâce à la production réelle de l'établissement qui vont alimenter la production future. Le développement de nouvelles activités, ou la reconversion de celles existantes, repose ainsi sur la capacité de l'établissement à autofinancer son activité.

L'ensemble de ces réformes s'inscrit dans la ligne du nouveau management public (Osborne D. et Gaebler T., 1992, Pollitt C., 1990) visant à transposer les méthodes et outils du management de l'entreprise au secteur public, jugé inefficace, bureaucratique, rigide, coûteux, non innovant et ayant une hiérarchie trop centralisée (Amar A. et Berthier L., 2006).

La réussite liée à la construction de ce dispositif de planification, tout comme le mouvement de responsabilisation, reposent en grande partie sur la possession d'informations exhaustives sur la production hospitalière, que ce soit en termes de facteurs de production mis en œuvre, ou encore de nature, de qualité et de quantité des outputs. Longtemps non satisfait en raison de la limitation des systèmes d'information aux quatre piliers historiques bien connus des gestionnaires du secteur sous le sobriquet des «quatre vieux»- le nombre d'admissions (entrées ou venues), le nombre de journées, le taux d'occupation, et la Durée moyenne de séjour (DMS) - (Engel F., Kletz F., Moisdon J.-C., et al., 2000, Moisdon J.-C. et Tonneau D., 1996), ce besoin d'information indispensable à des décisions de régulation pertinentes a trouvé une réponse au travers de l'adaptation du système américain des DRG (Diagnosis Related Groups) de description et de classification des séjours hospitaliers sous la forme du Programme de médicalisation des systèmes d'information (PMSI) à partir de 1982 (De Pouvourville G., Jeunemaitre A. et Condamin F., 1988 ; Rodrigues J.-M. et Goldberg S., 1984). En effet, le perfectionnement constant de ce système depuis sa généralisation en 1989 aux établissements hospitaliers publics et privés de court séjour, et son extension progressive aux autres champs du soin (soins de suite et de réadaptation, hospitalisation à domicile) autorisent désormais une connaissance extrêmement fine de la production hospitalière, connaissance à la disposition de l'ensemble des acteurs, établissements comme financeurs, puisque ces données sont de nature publique.

\section{Le PMSI, clé de voûte de l'information médico-économique}

Inspiré d'un système élaboré aux États-Unis à l'université de Yale dans les années 70 (Fetter R.B., Averill R., Brand D., et al., 1991, Fetter R.B. et Freeman J.L., 1986), le PMSI constitue aujourd'hui la clé de voûte du système d'information hospitalier français. Rendu obligatoire pour tous les établissements publics et privés de court séjour par la loi hospitalière de juillet $1991^{2}$, ce système vise à décrire de façon normalisée la production hospitalière française.

\footnotetext{
${ }^{2}$ Loi n91-748 du 31 juillet 1991 portant réforme hospitalière.
} 


\subsection{Constitution du PMSI et principes de fonctionnement}

La description par le PMSI de l'activité des établissements de santé de médecine, chirurgie et obstétrique (MCO) repose sur une convention de représentation de la production hospitalière au travers d'une nomenclature volontairement restreinte de familles de séjours nommées Groupes homogènes de malades $(\mathrm{GHM})^{3}$. La construction de ces groupes de séjours repose sur des analyses statistiques fondées principalement sur trois informations : le ou les diagnostics expliquant la prise en charge, les actes éventuels effectués lors de celle-ci, et un marqueur caractérisant le patient, principalement son âge. Partant de ces trois types de variables, le système classe automatiquement chaque séjour dans un groupe présentant statistiquement une homogénéité médico-économique. L'homogénéité médicale repose essentiellement sur la détermination du diagnostic principal de la prise en charge permettant d'orienter le séjour dans l'une des vingt-sept catégories majeure de diagnostic (CMD) ${ }^{4}$ chapotant les GHM, tandis que l'homogénéité économique est, quant à elle, établie par la mesure du pouvoir explicatif des trois types d'informations médicales sur une variable dépendante représentative du coût du séjour, originellement et encore en grande partie approché par la durée du séjour.

L'organisation actuelle du dispositif PMSI est le fruit de vingt-cinq ans d'évolutions, de choix (ou de non-choix), à la fois sur le contenu technique du dispositif et sur la visée de celui-ci en termes d'utilisation. Ces choix ont fait du PMSI, le fruit de compromis sociaux et techniques qui l'ont orienté dès la deuxième moitié des années 80 vers une finalité d'allocation budgétaire. Cette dernière s'est progressivement opérationnalisée à partir de 1994, à travers l'expérimentation Languedoc-Roussillon, jusqu'à atteindre sa pleine effectivité avec l'instauration de la Tarification à l'activité (T2A) (Capgras J.-B., 2009). En effet, depuis 2004, la première partie descriptive du PMSI a été adossée à une seconde permettant d'en faire le socle d'un paiement prospectif de l'activité des établissements de santé. Ce deuxième volet que constitue la T2A adjoint ainsi un tarif à chacun des GHM de la nomenclature nationale. Depuis 2008, le financement des établissements de santé MCO publics et privés est directement fondé sur leur activité décrite au travers des GHM et de leurs tarifs associés.

Le calcul de ce tarif repose sur l'analyse des coûts complets des séjours hospitaliers et des GHM dans lesquels ils se classent, estimés à partir de la comptabilité analytique d'une soixantaine d'établissements publics et privés participant sur la base du volontariat à l'Étude nationale des coûts (ENC) conduite sous l'égide de l'Agence technique de l'information sur l'hospitalisation (ATIH) (ATIH, 2007). À partir de ces coûts, des tarifs bruts sont calculés, puis pondérés afin d'obtenir les tarifs définitifs en fonction, d'une part, de l'enveloppe globale de financement dédiée aux activités hospitalières dans le cadre annuel de l'Objectif national des dépenses d'assurance-maladie (ONDAM) ${ }^{5}$ et d'autre part, des priorités des pouvoirs publics en termes de santé publique.

\footnotetext{
${ }^{3}$ Les vingt-deux millions de séjours annuellement pris en charge par les établissements de santé de court séjour sont ainsi représentés à travers environ 2300 GHM aujourd'hui contre 400 lors du lancement du PMSI.

${ }^{4}$ Chaque GHM est ainsi lui même classé dans une seule et unique CMD reposant sur une signification clinique généralement liée à un organe (affection du tube digestif) ou, plus rarement, à un groupe étiologique (maladies liées à une infection au VIH) ou un type de prise en charge (séances).

${ }^{5}$ L'ONDAM est voté chaque année par le Parlement dans le cadre de la loi de financement de la sécurité sociale.
} 
Les différentes informations recueillies au niveau du PMSI peuvent être distinguées en trois types :

- les informations administratives principales sont le numéro FINESS identifiant l'établissement ayant pris en charge le séjour, le nombre d'unités médicales qu'a fréquenté le patient au cours de son séjour, les modes d'entrée et de sortie du séjour (entrée simple, entrée par les urgences, transfert depuis un autre établissement et bien sûr décès le cas échéant pour la sortie), la provenance et la destination du patient (domicile, autre établissement de santé ou médicosocial), la durée du séjour ainsi que le mois et l'année de sortie et enfin l'âge et le sexe du patient;

- les informations médicales sont essentiellement constituées par le diagnostic principal ayant motivé la prise en charge du patient ainsi que par les éventuels diagnostics associés et actes réalisés au cours du séjour;

- enfin, les informations de groupage mentionnent principalement le GHM et la CMD dans lesquels est classé le séjour ainsi que le tarif qui lui est associé dans le cadre de la T2A.

Ces informations, rassemblées sous formes de fichiers informatiques comportant plus d'une centaine de variables, constituent une véritable mine d'informations pour les observateurs et les gestionnaires du système hospitalier.

\subsection{Une information quasi exhaustive et qualitativement contrôlée}

L'un des intérêts du PMSI réside dans la publicité et l'exhaustivité des données disponibles. Alors que dans la plupart des domaines d'activité économique, les données sur le marché sont à la fois rares, limitées à des segments ou des niches, payantes, souvent exclusives, et mises à jour de façon relativement espacée (au mieux l'année pour les études de marché les plus performantes), le PMSI constitue en effet une source d'informations publique et continue, accessible à tous les producteurs comme aux patients et aux financeurs, qui offre une description complète et très précise des activités réalisées sur un territoire donné, dans une perspective à la fois synchronique et diachronique. Dans plusieurs régions, ces données sont gracieusement distribuées aux établissements par l'ARS, soit sous la forme de données retraitées, soit directement sous la forme d'une base régionale des fichiers PMSI. Par ailleurs, les nouvelles modalités tarifaires fixées par l'ATIH en 2009 pour l'obtention des bases de données constitutives du dispositif PMSI accordent ces dernières gratuitement, hors frais de traitement, aux établissements de santé ${ }^{\text {. }}$

Le lien entre financement des établissements et information sur l'activité constitue un incitatif puissant pour les établissements à renseigner correctement le PMSI, et est par conséquent un facteur d'amélioration de la qualité et de l'exhaustivité des données qu'il recense. En particulier l'application de la T2A à partir de 2004 a constitué un véritable moteur du renseignement du PMSI par les établissements de santé. La visée budgétaire du PMSI a entraîné une amélioration du codage de l'activité hospitalière en créant un équilibre entre deux incitatifs opposés. D'un côté, soumis à la T2A, les établissements sont incités à coder toute activité ayant mobilisé des ressources, et d'un autre côté, l'Assurance-maladie est incitée à contrôler les éventuels surcodages afin de limiter les paiements non justifiés ${ }^{7}$.

\footnotetext{
${ }^{6}$ Délibération $n^{\circ} 4$ du 5 mai 2009 du conseil d'administration de l'Agence technique de l'information sur I'hospitalisation, BO Santé, Protection sociale, Solidarité n²009/5 du 15 juin 2009.

${ }^{7}$ Le contrôle de l'assurance maladie s'opère en deux phases. Une première étape consiste à identifier statistiquement les codages déviant et une deuxième étape procède par vérification sur site de la justification du codage par rapport aux éléments présents dans les dossiers patient ciblés à la première étape.
} 
La finalité d'allocation de ressource du PMSI conditionne cependant fortement la nature des informations recueillies dans le dispositif. Les évolutions successives du PMSI ont par la suite toujours privilégié cette visée budgétaire. Par exemple, dans le cadre de la planification des activités de cancérologie par territoire, l'identification des activités de cancérologie est limitée par le système de classification des séjours existant, alors qu'il aurait pu être imaginé une évolution de ce dernier dans le but d'améliorer son pouvoir descriptif de l'activité de cancérologie, certes aux dépens du respect de l'homogénéité médico-économique du système (Titton M., Binquet C., Vourc'h M., et al., 2008). De plus, du fait du lien entre allocation budgétaire et description de l'activité, l'information recueillie au sein du PMSI par les établissements se limite généralement aux informations impactant le classement dans un groupe tarifaire entraînant un niveau de renseignement des autres champs très faible, voire inexistant.

\subsection{Un système fortement mobilisé par l'ensemble des acteurs}

Les différents usages du PMSI peuvent s'inscrire dans trois grandes finalités gestionnaires : l'allocation budgétaire concrétisée par le système de tarification à l'activité présenté précédemment, la planification par les tutelles, la gestion interne aux établissements. Le schéma ci-après présente dans une perspective chronologique les différents outils et usages correspondant à ces trois finalités (cf. Figure 1).

\section{Figure 1 - Chronologie de la mise en œuvre des informations issues du PMSI à des fins de gestion par les établissements de santé et les tutelles.}
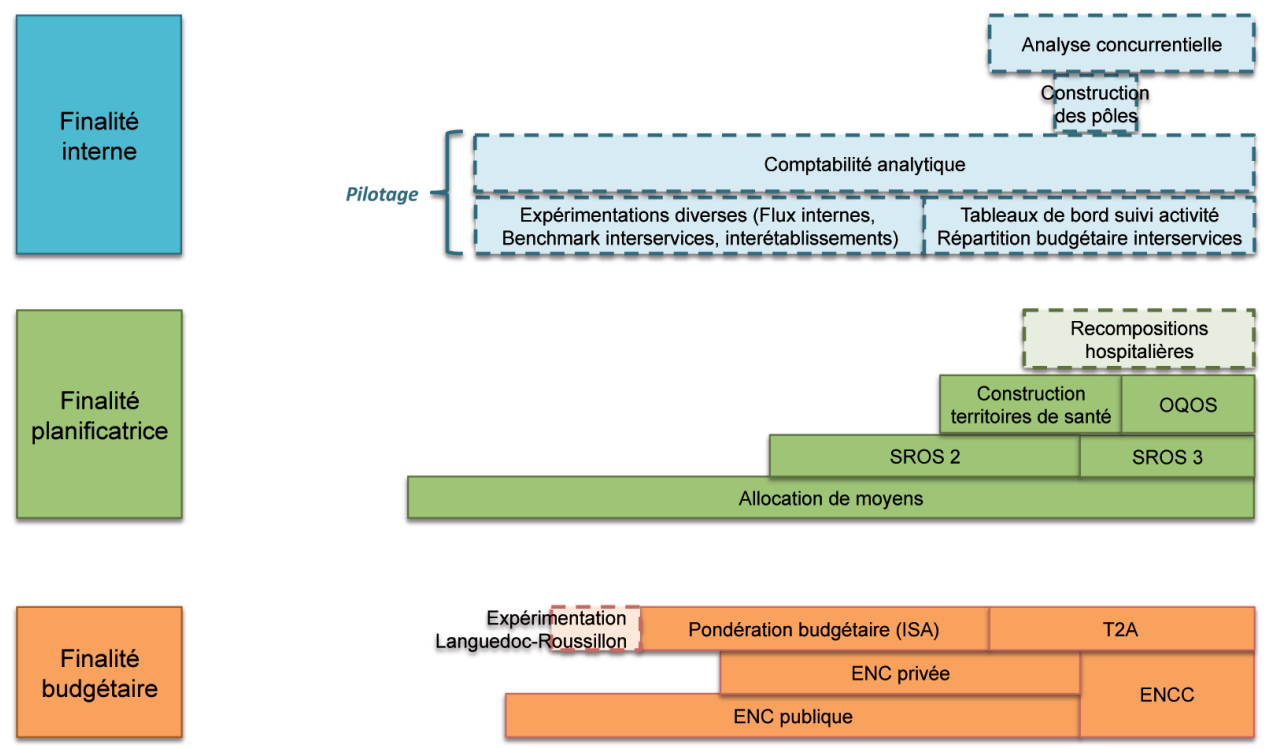

Information

\begin{tabular}{|l|l|l|l|l|l|l|l|l|l|l|l|l|l|l|l|l|l|l|l|l|l|l|l|l|l|l|l|}
\hline 82 & 83 & 84 & 85 & 86 & 87 & 88 & 89 & 90 & 91 & 92 & 93 & 94 & 95 & 96 & 97 & 98 & 99 & 00 & 01 & 02 & 03 & 04 & 05 & 06 & 07 & 08 & 09 \\
\hline
\end{tabular}

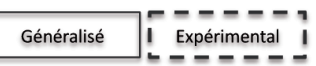


Si l'utilisation première du PMSI relève d'une finalité budgétaire, les pouvoirs publics se sont également emparés de ce système d'information pour répondre à leur mission de planificateur/régulateur de l'offre de soins. En premier lieu, l'analyse de l'activité que permet le PMSI a servi de support à l'allocation de moyens en termes financier, en lien direct avec la finalité budgétaire, mais également en termes de délivrance d'autorisations concernant, par exemple, l'exercice de la chirurgie oncologique ${ }^{8}$. L'élaboration des Schéma régionaux d'organisation sanitaire de première et deuxième générations (SROS 1 et 2) a reposé en grande partie sur l'analyse de l'activité des établissements de santé tant en ce qui concerne la recherche de complémentarités inter-établissements qu'en ce qui concerne la délimitation de zones de recours aux soins (Lernout T., Lebrun L. et Bréchat P.-H., 2007). En particulier, l'étude des flux de patients entre leur lieu de résidence et l'établissement de prise en charge a fait l'objet de nombreux travaux afin de définir les territoires de santé, supports de la planification régionale dans le cadre du SROS 3 (Coldefy M. et Lucas-Gabrielli V., 2008, Haas S. et Vigneron E., 2004). La mise en place des Objectifs quantifiés de l'offre de soins (OQOS) courant 2006, dont l'objet est de fixer des seuils et plafonds d'activité par discipline, par territoire et par établissement, repose également sur cette source d'information, alors mobilisée à la fois comme base projective et outil d'évaluation/contrôle du respect des objectifs préalablement fixés (Pereira C. et Ravissot S., 2007). Enfin, de façon moins généralisée, certaines ARH ont pu mobiliser le PMSI en appui de leurs réflexions quant au regroupement de certaines activités, voire établissements, dans le cadre des nombreuses opérations de recomposition hospitalière conduites au cours de la dernière décennie (Claveranne J.-P., Pascal C., Capgras J.-B., et al., 2006, Haas S. et Vigneron E., 2009). L'intégration des codes géographiques de résidence des patients hospitalisés à partir de 1999 a permis leurs mobilisations dans le cadre de l'élaboration des SROS 2 par les ARH (Aublet-Cuvelier B., 2003).

Les autorités de tutelles n'ont cependant pas été les seules à s'emparer de ce gisement d'information. Nombre d'établissements de santé, publics et privés, ont eux aussi vu un intérêt à mobiliser le PMSI à des fins diverses de gestion interne. C'est ainsi, que dès la généralisation du recueil de données PMSI, différentes expérimentations ont été développées par les établissements de santé et en particulier par les hôpitaux publics qui disposaient des compétences et des postes des médecins de la nouvelle spécialité « information médicale » créée pour traiter le PMSI ${ }^{9}$.

Les premières études concernant les flux de patients internes à l'établissement (flux inter-unités de soins) (Dellinger A. et Bolard P., 2010) et les premiers benchmarks d'activité interservices, voire inter-établissements (Coca E., 1998, Noury J.-F., 2000), ont ainsi été conduits dès les années 90 par certains établissements dans des démarches de réingénierie de leurs processus de prise en charge (Claveranne J.-P. et Pascal C., 2004). L'étude des flux internes de patients a également été mobilisée plus récemment par certains établissements afin de regrouper les différents services de soins en pôles d'activité instaurés par l'ordonnance

\footnotetext{
${ }^{8}$ Arrêté du 29 mars 2007 fixant les seuils d'activité minimale annuelle applicables à l'activité de soins de traitement du cancer; Circulaire DHOS/O/INCa du 26 mars 2008 relative à la méthodologie de mesure des seuils de certaines activités de soins de traitement du cancer.

${ }^{9}$ Circulaire DH/PMSI n³03 DU 24 juillet 1989 relative à la généralisation du programme de médicalisation des systèmes d'information et à l'organisation de l'information médicale dans les hôpitaux publics.
} 
de $2005^{10}$. Les établissements ont également développé divers outils de contrôle de gestion reposant sur les informations du PMSI tels les comptes de résultats par activité (CREA) ou encore les tableaux coûts/casemix (TCCM) qui mettent en correspondance les coûts par GHM de l'établissement avec les coûts pour la même activité relevés dans le cadre de l'Étude nationale des coûts (ENC) (Kern A., 2006). Enfin, l'accès de chaque établissement aux données PMSI des autres établissements a permis le développement, encore embryonnaire, d'études de marché et d'analyses concurrentielles souvent conduites par des équipes de recherche ou des consultants spécialisés pour le compte des établissements eux-mêmes ou pour celui de leur fédération ${ }^{11}$.

\subsection{Des usages en devenir : étude de marché et analyse de la concurrence}

Si les utilisations des informations du PMSI présentées supra sont désormais mobilisées par un nombre important d'établissements et de tutelles, le système offre encore un fort potentiel d'exploitation, seul ou combiné avec d'autres sources d'informations, financières ou géographiques par exemple. Ces nouveaux usages restent pour l'instant confinés à quelques expérimentations d'établissements et planificateurs avant-gardistes.

\subsubsection{L'étude de marché ou analyse concurrentielle}

Dans le cadre de différentes recherches-interventions, notre équipe a accompagné plusieurs établissements dans la réalisation d'analyse concurrentielle reposant sur l'utilisation des données issues du PMSI. Ainsi, l'origine géographique du patient (son lieu de résidence), les GHM, la valorisation de l'activité avec les coûts de l'ENC sont autant d'éléments mobilisables dans le cadre d'une analyse concurrentielle. Celle-ci se déroule généralement en trois temps : analyse du profil d'activité de l'établissement, délimitation de la zone de recrutement de l'établissement et enfin, calcul des parts de marché.

\section{- L'analyse du profil d'activité de l'établissement}

Le profil d'activité des établissements peut être étudié à partir de l'analyse de la répartition entre chaque GHM des séjours pris en charge. Ce niveau d'analyse peut cependant rapidement poser problème dans le cadre de l'identification des caractéristiques principales de l'activité du fait du nombre important de GHM différents. Les établissements peuvent alors se référer à des regroupements alternatifs dont le principe de fonctionnement repose sur le regroupement de GHM soit par organe (Appareil circulatoire, Système nerveux, etc.) soit par spécialité et moyens de production mobilisés (Orthopédie-traumatologie, Chirurgie digestive, etc.). Si, jusqu'à récemment, le regroupement le plus usité était l'outil d'analyse du PMSI (OAP), la dernière version de la classification introduite en 2009 a consacré le

\footnotetext{
${ }^{10}$ Ordonnance $n^{\circ} 2005-406$ du 2 mai 2005 simplifiant le régime juridique des établissements de santé.

11 Les établissements de santé adhèrent généralement à l'une des trois grandes fédérations chargées de représenter leurs intérêts auprès des pouvoirs publics : la Fédération hospitalière de France (FHF) pour les établissements publics, la Fédération des établissements hospitaliers et d'aide à la personne non lucratifs (FEHAP) et la Fédération des établissements privés (FHP).
} 
système des groupes d'activité ${ }^{12}$. En fonction des objectifs poursuivis et en associant les différents outils de classification, l'analyse peut recouper différents niveaux de regroupement/ segmentation : Catégories majeures de diagnostic (CMD), distinction activités médicale, chirurgicale et obstétricale, segmentation ambulatoire/hospitalisation complète, niveaux de sévérité, groupes d'activité, etc.

\section{Figure 2 - Exemple de représentation de l'activité d'un établissement par Segment d'activité OAP}

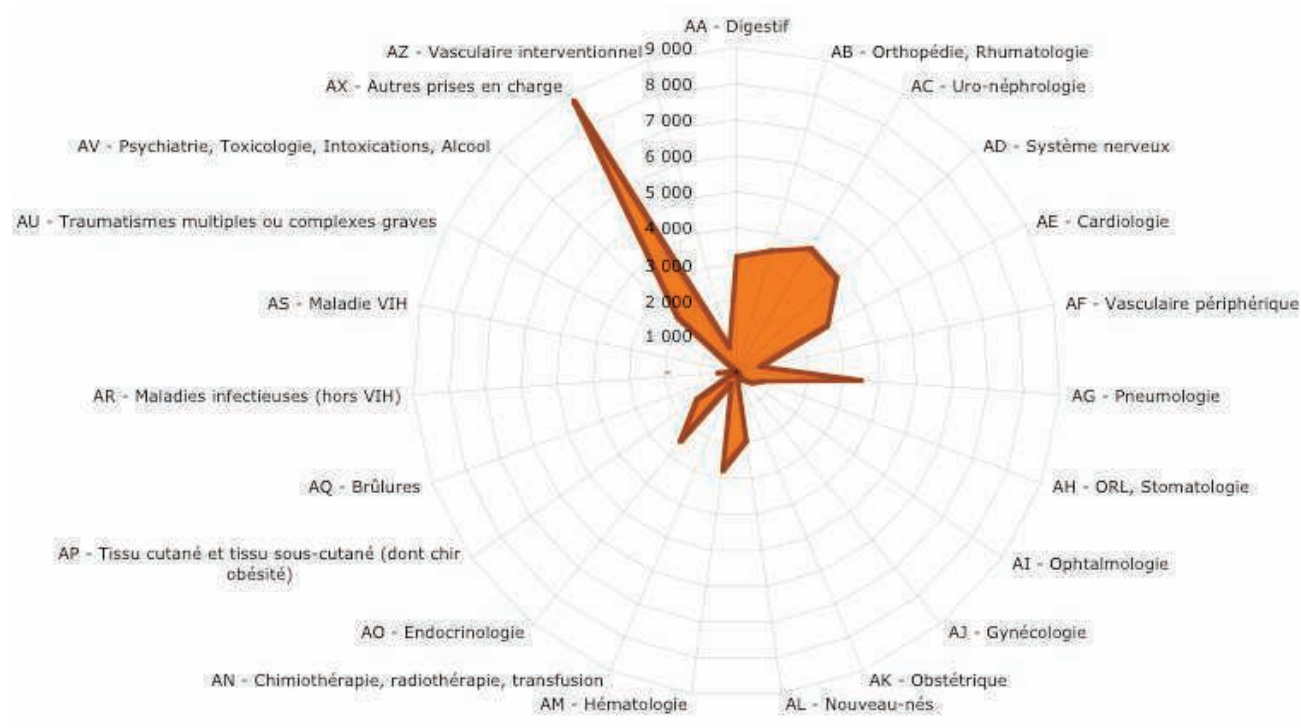

Figure 3 - Exemple d'analyse du profil d'activité MCO d'un établissement

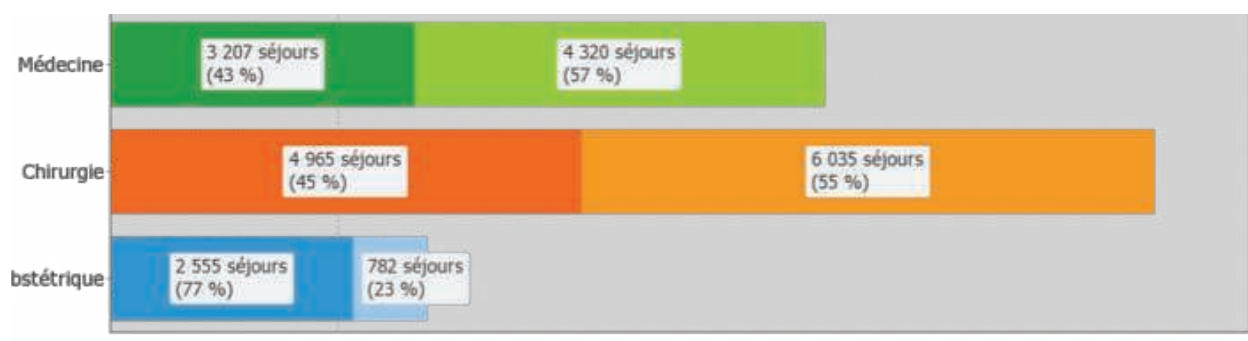

zurs erronés exclus : $0,000 \%$

\footnotetext{
${ }^{12}$ La classification en groupes d'activité est issue d'un travail conduit par la cellule d'analyse d'activité de l'Agence technique de l'information sur l'hospitalisation (ATIH). II repose sur la mise en commun des caractéristiques du regroupement OAP et d'un autre regroupement réalisé par le Dr Ruiz du Comité technique régional de l'information médicale (COTRIM) de Midi-Pyrénées.
} 


\section{- La délimitation de la zone d'étude}

Dans le cadre de l'analyse du profil d'activité, l'ensemble de l'activité prise en charge par l'établissement, sans limite géographique concernant le lieu de résidence des patients, est étudié. Or, la question de la délimitation de la zone de recrutement est essentielle à la fois à la détermination des parts de marché et à l'identification des concurrents. Au premier abord, on pourrait être tenté de définir comme appartenant à la zone de recrutement de l'établissement, toute zone géographique du PMSI dont au moins l'un des habitants a été pris en charge par l'établissement en question. Cette solution, si elle présente l'avantage de l'exhaustivité ne se révèle cependant pas satisfaisante dans la pratique pour diverses raisons. La principale d'entre elles est liée à la nature du renseignement de l'information géographique au sein du PMSI. La zone géographique ${ }^{13}$ d'origine du séjour correspond à la zone géographique de résidence du patient. La plupart du temps cela ne pose aucune difficulté mais dans un certain nombre de cas non négligeable cela contribue à perturber l'identification de la zone de recrutement. En particulier, le cas des touristes hospitalisés sur leur lieu de villégiature est emblématique de cette difficulté.

La solution consiste alors à ne prendre en compte que les x premiers pour cent des séjours, focalisant ainsi l'attention sur les zones géographiques les plus pourvoyeuses de patients. En marketing, ce taux est généralement fixé à $50 \%$ afin de minimiser le coût de la collecte d'information (Boulocher V., Flambard S. et Jean S., 2006), qui est inexistant dans le cadre du PMSI dont les données ont déjà été recensées à des fins d'allocation budgétaire. Dans l'application en ligne SNATIH ${ }^{14}$ de traitement des données d'activité et de benchmark développée par l'ATIH, le taux est fixé à $80 \%$. Différents tests sous contrainte et études empiriques ont cependant montré qu'un seuil de $90 \%$ excluait moins les zones géographiques peu peuplées (donc générant logiquement moins de séjours) mais ayant pourtant légitimement leur place dans la zone de recrutement, surtout si la zone de recrutement est caractérisée par de fortes disparités en termes de densité de population (Pascal C., Capgras J.-B., Botton J.-F., et al., 2009).

\section{- La détermination des parts de marché}

En fonction des objectifs poursuivis, les parts de marché peuvent être déterminées pour chaque niveau de segmentation de l'activité retenu et pour l'ensemble de la zone de recrutement ou pour chacune des zones géographiques la composant.

\footnotetext{
${ }^{13}$ La zone géographique du PMSI correspond dans la majorité des cas à la zone postale sauf pour les zones postales comprenant moins de mille habitants qui font alors l'objet d'un regroupement.

${ }^{14}$ Système national d'information sur l'hospitalisation à destination des Conseillers généraux des établissements de santé du ministère en charge de la santé.
} 
Figure 4 - Exemple de délimitation d'une zone de recrutement par étude de l'origine des séjours

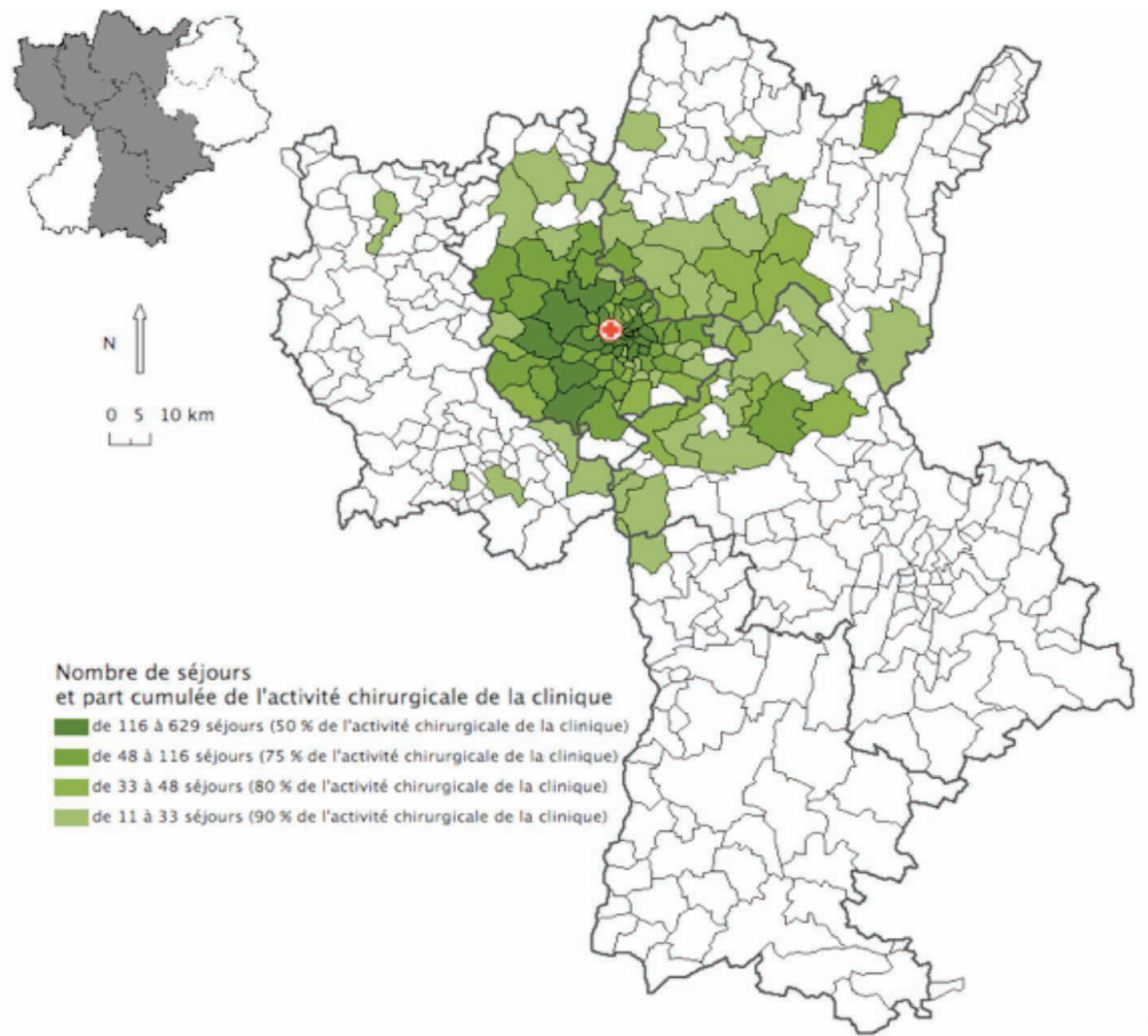

Figure 5 - Exemple de détermination des parts de marché par zone géographique

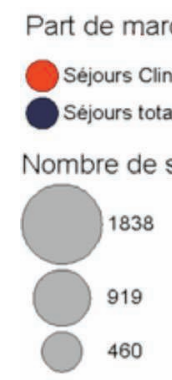

que

Nombre de séjours

Taux de recrutement

$\square$ Plus de $25 \%$

de $20 \%$ a $25 \%$ $\square$ de $15 \%$ à $20 \%$ $\square$ Moins de $15 \%$

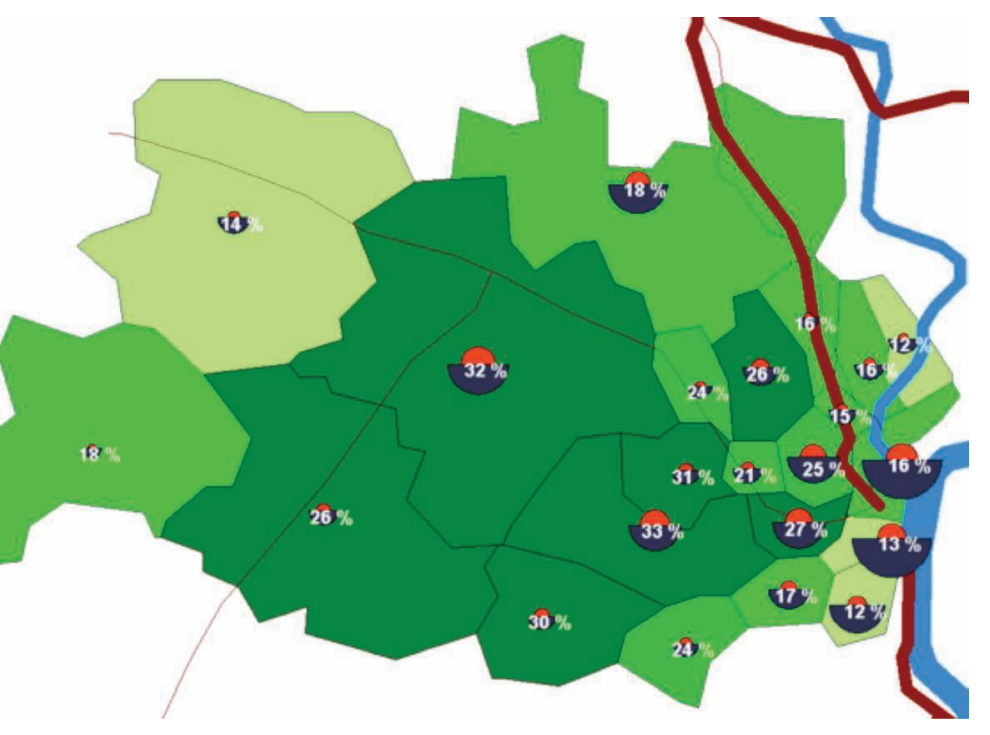


Une fois les parts de marché déterminées, l'établissement peut être positionné par rapport à ses concurrents et l'évolution de ce positionnement suivi chaque année.

\section{Figure 6 - Exemple de positionnement de la Clinique T par rapport aux autres établissements intervenant sur sa zone de recrutement (Urologie, ambulatoire/ hospitalisation complète)}

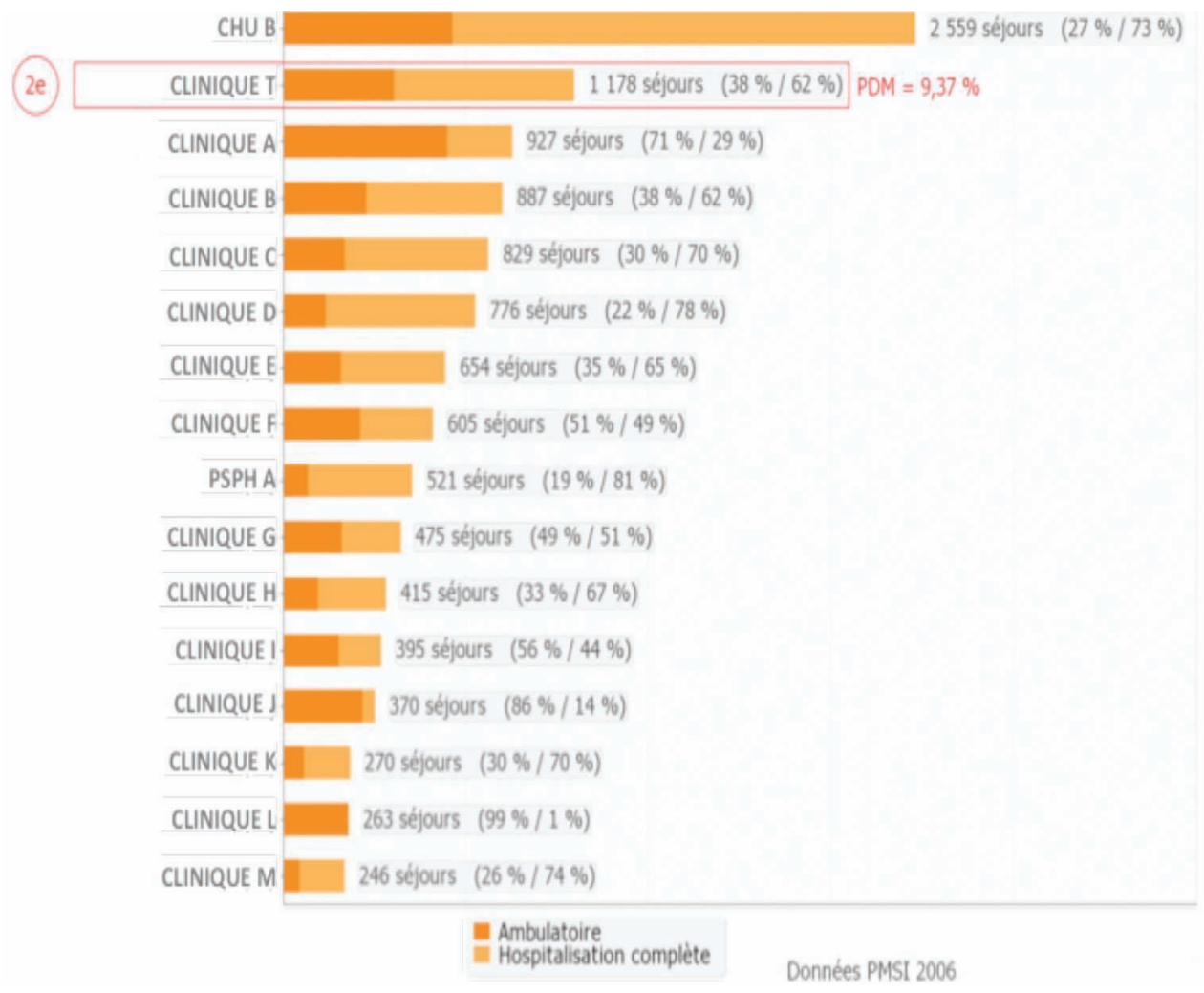

Le positionnement peut reposer non seulement sur des données quantitatives comme le volume d'activité ou le chiffre d'affaires produit mais également sur des aspects plus qualitatifs comme la lourdeur moyenne des cas traités.

\subsubsection{L'analyse de l'intensité concurrentielle}

Placé à un niveau meso de gestion de territoires, à la fois par les tutelles et par les acteurs locaux eux-mêmes, le PMSI offre une représentation de l'activité hospitalière MCO permettant des niveaux d'analyses variés, tant du point de vue de la qualification médicale de l'activité, que de la catégorisation géographique. La mobilisation des données du PMSI enrichies d'informations administratives, démographiques et géographiques, permet par exemple de décrire les comportements de recours aux soins des patients aux travers d'indicateurs de concentration issus de l'économie industrielle. Un des moyens privilégiés d'objectiver la concurrence en économie industrielle est de mesurer la concentration des 
acteurs sur les éléments objets de la concurrence (séjours, ressources, etc.). Si le dénombrement des concurrents fournit des premiers éléments frustres d'analyse, des indices dits de « concentration concurrentielle» sont également largement mobilisés. Ces indices qui répondent à un certain nombre de propriétés mathématiques (Hall M. et Tideman N., 1967) constituent une mesure indirecte de la concurrence reposant sur la corrélation existant entre la concentration et la notion de pouvoir de monopole (Miller J.P., 1955, Saving T.R., 1970, Scitovsky T., 1955).

Parmi ces différents indices, l'indice de Herfindahl-Hirschmann (HHI) est l'indice de concentration absolue le plus utilisé, que ce soit par les autorités de contrôle de la concurrence européennes et américaines (Linda R., 1976) mais également dans le cadre de très nombreux travaux de recherche de tous secteurs (Dobruszkes F., 2007), y compris dans le secteur hospitalier (Capps C.S., Dranove D., Greenstein S., et al., 2002; Schramm C.J. et Renn S.C., 1984).

Le HHI est la somme des carrés des parts de marché de l'ensemble des acteurs du secteur, avec $\mathrm{p}_{\mathrm{j}}$ pour les parts de marché et $\mathrm{n}$ le nombre d'acteurs (Herfindahl O.C., 1950; Hirschman A.O., 1945). L'indice prend des valeurs comprises entre $1 / \mathrm{n}$ et 1 . Plus le HHI est proche de 1, plus le marché est concentré et plus le HHI est proche de $1 / \mathrm{n}$, plus le marché est éclaté et la concurrence importante.

L'illustration qui suit applique ce type de mesure aux données issues du PMSI sur la région Rhône-Alpes en pneumologie médicale, activité à la fois nécessaire sur tout le territoire et représentant, avec près de $5 \%$ de l'activité hospitalière de court séjour, la troisième discipline médicale la plus importante. À partir des données d'activité issues du PMSI, les parts de marché et le HHI ont été calculés pour chacune des 517 zones géographiques composant la région Rhône-Alpes. Cette étude révèle que l'activité de pneumologie médicale est particulièrement concentrée avec un HHI moyen situé entre 0,4 et 0,5 . Ainsi, près de $43 \%$ de la population rhônalpine réside dans une zone caractérisée par une structuration allant du duopole au monopole en pneumologie médicale ${ }^{15}$.

\footnotetext{
${ }^{15}$ Transcris en nombre d'établissements équivalents, un $\mathrm{HHI}$ situé entre 0,4 et 0,5 correspond à une activité partagée entre deux établissements.
} 


\section{Carte 1 - HHI par zone géographique — Pneumologie médicale}

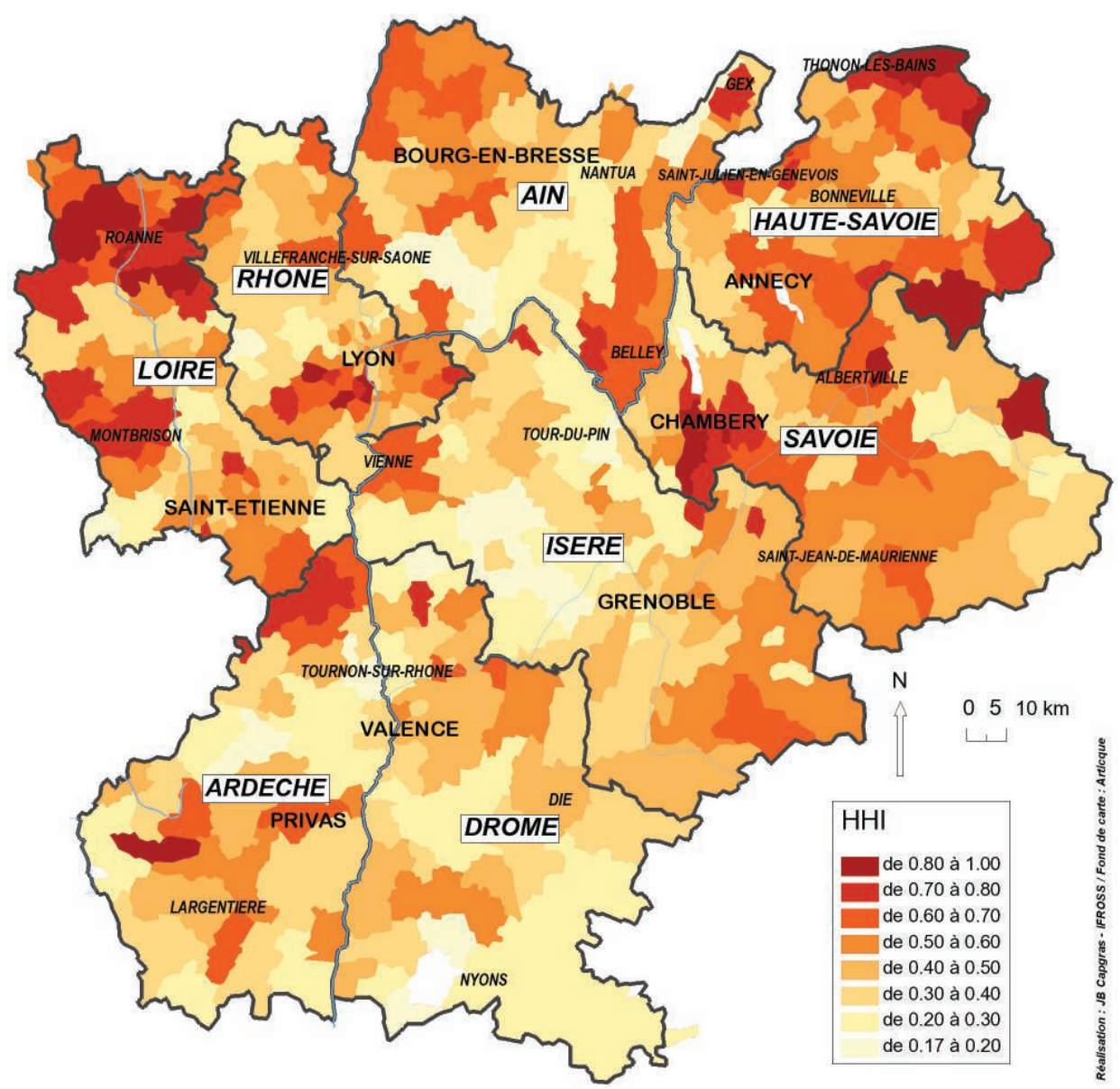

Les zones les plus concentrées apparaissent en foncé sur la carte. Dans cette illustration sur la pneumologie médicale, elles se situent autour de Roanne, de Chambéry, de Thononles-Bains, de Montbrison et de Sallanches.

Le recoupement des informations sur la concentration des activités avec des données démographiques et géographiques permet de compléter l'analyse, notamment au niveau des zones de recrutement (cf. Tableau 1). 
Tableau 1 - Caractéristiques des zones de recrutement rhônalpines en pneumologie médicale

\begin{tabular}{|c|c|}
\hline \multirow{2}{*}{ Nombre d'établissements rhônalpins exerçant l'activité } & Pneumologie médicale \\
\hline Surface moyenne de la ZR & 18 \\
\hline Population moyenne de la ZR & 395977 \\
\hline Densité moyenne de population par ZR (hab./km²) & 315,5 \\
\hline Taux d'urbanisation' moyen & $83,6 \%$ \\
\hline Nb moyen de séjours issus de la ZR & 651 \\
\hline HHI max & 0,98 \\
\hline HHI moyen & 0,39 \\
\hline HHI minimum & 0,05 \\
\hline
\end{tabular}

S'appuyant sur des analyses de ce type, le décideur hospitalier et/ou la tutelle peuvent affiner leur approche de l'offre de soins, notamment en prenant en compte la répartition des activités sur un territoire donné et en considérant un niveau infra-hospitalier.

\section{De l'information à l'action : I'impossible passage?}

Les utilisations possibles du PMSI décrites précédemment montrent à la fois le potentiel de l'information médico-économique délivrée par le PMSI dans la décision stratégique des établissements publics et des régulateurs, et le processus de mobilisation entamé par tous ces acteurs autour de l'outil. Ainsi, alors que la principale caractéristique du système hospitalier est souvent présentée, notamment dans le cadre du mainstream économique de la théorie de l'agence (Naegelen F. et Mougeot M., 1998), comme étant celle d'un manque d'information disponible, dans quel autre secteur d'activité une entreprise (ici un établissement) peut-il avoir accès aux caractéristiques, client par client (ici patient par patient), des prestations fournies et du client en question (patient) pour l'ensemble des autres entreprises (établissements) du secteur?

La question qui se pose alors est celle des leviers d'action à disposition des autorités de régulation et des directions hospitalières publiques pour agir sur l'offre à partir des analyses issues de ces informations médico-économiques.

\subsection{Entre marché et régulation : les paradoxes du régulateur}

Les Agences régionales de santé, créées par la loi n 2009-879 du 21 juillet 2009 dite loi «Hôpital, patients, santé et territoires », qui regroupent et remplacent les sept organismes autrefois en charge des politiques de santé dans les régions et les départements ${ }^{16}$ constituent

\footnotetext{
${ }^{16}$ Agence régionale de I'hospitalisation, Direction régionale des affaires sanitaires et sociales, Direction départementale des affaires sanitaires et sociales, Caisse régionale d'assurance-maladie, Union régionale des caisses régionales d'assurance-maladie, Groupement régional de santé publique, Mission régionale de santé.
} 
désormais le pilote unique de la régulation du système sanitaire au niveau régional. Elles ont pour mission de réguler, orienter et organiser l'offre de services de santé, de manière à répondre aux besoins en matière de soins et de services médico-sociaux, et à garantir l'efficience du système de santé. Cet objectif d'optimisation de l'offre de soins les conduit à promouvoir la complémentarité entre établissements aux dépens de la concurrence, conçue comme source d'inefficience au niveau du territoire.

Ce système vise, selon ces concepteurs, à inciter les hôpitaux publics, jugés peu productifs, à augmenter leur activité, en profitant des marges de manœuvre liées à l'asymétrie d'information sur leur bassin d'activité (Cash R., 2004). Le principe libéral porté par cette réforme apparaît comme antinomique avec les incitations à la mutualisation des moyens et de l'activité émanant du planificateur : la mutualisation de l'activité entre établissements d'un même territoire, au sein d'un groupement de coopération sanitaire par exemple, se solde non pas par une augmentation de l'activité et du chiffre d'affaires de chaque membre, mais par un partage de l'activité totale entre ces derniers, générant des pertes de revenus pour les uns et des gains pour les autres. Ces gains et ces pertes sont d'autant plus importants que la plupart des territoires sont organisés sous forme de duopoles ou d'oligopoles restreints.

De même, la recherche de l'augmentation de la vitesse de rotation des patients sous l'effet de la T2A est au cœur de l'incohérence des filières amont-aval, puisqu'elle incite les hôpitaux et les cliniques à réduire leur durée moyenne de séjour, reportant ainsi sur les établissements d'aval, ou sur la médecine de ville, les coûts de prise en charge, sans effet global sur le système (Hirtzlin I., 2007).

Les objectifs quantifiés de l'offre de soins (OQOS), instaurés dans chaque territoire pour limiter les effets pervers de la T2A en encadrant la production de chaque établissement, démontrent les limites de leur efficacité. Les dépassements d'objectifs sont exceptionnellement sanctionnés, et donnent le plus souvent lieu à une révision à la hausse de l'objectif pour l'année suivante. Cette clémence du planificateur trouve en grande partie sa justification dans l'impossibilité pratique de bâtir sur cinq ans des prévisions d'activités réalistes : le départ d'un médecin vers un autre établissement, la suppression d'une activité voire la fermeture d'une clinique suite à des difficultés économiques sont autant d'événements sur lesquels l'ARS n'a ni prise légale, ni réelle capacité d'anticipation, et qui, pourtant, modifient profondément la structure de l'offre locale. Les objectifs quantifiés ne peuvent dans ce contexte être raisonnablement opposés aux hôpitaux qui doivent accroître leur activité pour s'adapter à des évolutions imprévues.

Plus largement, ce mécanisme se heurte également - au moins dans les grandes agglomérations dans lesquelles l'offre hospitalière est abondante - à la liberté de choix du patient, qui constitue l'un des fondements du système de santé français : puisque tout patient a le droit de choisir son médecin et par conséquent son établissement au sein d'un territoire, comment l'autorité publique peut-elle opposer un objectif quantifié à un établissement et donc à un patient?

À la lecture de ce qui précède, on comprend que la tarification à l'activité et l'intensité des comportements concurrentiels qu'elle provoque entre établissements au sein des territoires (Lehtonen T., 2007) affaiblissent considérablement les capacités du régulateur à effectuer une véritable régulation. 


\subsection{De la décision à l'action : la stratégie désarmée des hôpitaux publics}

L'introduction de la T2A, conjuguée aux mesures prises pour renforcer la responsabilisation et l'autonomisation des établissements, notamment au travers de la contractualisation pluriannuelle avec les agences régionales de santé, a accéléré la tentation des directions hospitalières de se doter d'une réelle autonomie stratégique vis-à-vis de la tutelle.

Ce désir d'autonomie se manifeste par le développement ou l'acquisition d'outils de diagnostic stratégique, ainsi que de compétences dédiées : la plupart des hôpitaux disposent ainsi d'une direction de la stratégie, et se dotent de contrôleurs de gestion. Les données du PMSI, utilisées seules ou combinées avec d'autres informations de nature financière (comptabilité analytique de l'établissement, données de l'Étude nationale des coûts, base de comparaison des coûts hospitaliers d'Angers), démographique (données INSEE), ou encore administrative (fichier des médecins adresseurs et des établissements d'amont et d'aval), constituent la source principale de ce travail de diagnostic.

En fonction des situations particulières, ces données aboutissent le plus souvent à dégager des stratégies portant sur 3 dimensions :

- L'augmentation du volume d'activité, à structure d'activité inchangée ;

- la réorganisation du portefeuille d'activités;

- La réduction des coûts de production.

L'augmentation du volume d'activité suppose l'existence de gisements de productivité et d'un potentiel de recrutement de patients supplémentaires. La réorganisation du portefeuille consiste quant à elle à concentrer la production sur les créneaux les plus profitables, soit en sélectionnant les patients, soit en créant de nouvelles activités. Elle passe aussi par une réflexion sur les éventuelles alliances à sceller avec d'autres établissements publics ou privés pour se répartir ou se partager certaines activités. Ces stratégies nécessitent d'une part une analyse détaillée de la répartition par segments d'activités de la production, conjuguée à l'examen des zones de chalandise, des réseaux d'adresseurs, et des évolutions de l'état de santé de la population, et d'autre part le calcul de coûts de production, afin d'estimer un taux de marge.

La réduction des coûts de production peut se jouer dans deux directions :

- la baisse des frais fixes : elle est à rechercher dans une autre organisation interne de l'hôpital, et notamment dans ce que l'industrie a appelé le downsizing (Carter T., 1999, Tomasko R.M., 1987), c'est-à-dire une recherche systématique de réduction de la taille et des niveaux hiérarchiques des unités de production dans l'objectif d'une plus grande efficience et d'une plus grande flexibilité. Une telle logique passe par exemple par un ajustement des capacités du plateau technique et d'hébergement à la réalité de la production hospitalière (Gabel R.A., 1999) et non plus aux exigences de confort des médecins ou des personnels. Si l'on prend comme référence une production donnée de 1500 interventions annuelle par bloc opératoire, on peut aisément évaluer les économies à réaliser dans de nombreux hôpitaux pour lesquels les taux d'occupation sont bien souvent inférieurs à $50 \%$.

- la baisse des charges variables : cette diminution passe par une analyse des processus et la poursuite des démarches qualité engagées dans le sillage de l'instauration de l'obligation de certification des établissements de santé depuis les ordonnances Juppé d'avril 1996. 
La mise en œuvre de ces stratégies, qui reposent majoritairement sur les données issues du PMSI, se heurte cependant à trois séries d'obstacles.

1. Le premier - et sans doute le plus important - est la raréfaction de la ressource médicale. Choisir ses activités nécessite de disposer des ressources nécessaires à ce choix, notamment d'avoir les moyens d'embaucher de nouveaux professionnels médicaux ou de se séparer d'autres, tant d'un point de vue réglementaire que financier. On comprend dès lors que cette stratégie est difficilement envisageable à l'heure actuelle compte tenu de la rigidité des statuts hospitaliers et surtout de la démographie médicale, qui place les praticiens en situation de choisir leur lieu d'exercice. La situation est particulièrement préoccupante pour la chirurgie : alors que, au 1er janvier 2007, seuls 31,3\% des chirurgiens exercent à l'hôpital public, d'ici début 2019, les effectifs actuels des praticiens hospitaliers en chirurgie devraient diminuer de près de $30 \%$ en raison des départs à la retraite prévus (Milon A., 2008).

La fuite des médecins de l'hôpital vers la clinique privée dans certaines spécialités comme la cardiologie ou la radiologie s'oppose également à la réduction des coûts, puisqu'elle contraint l'hôpital à supporter les surcoûts liés à l'amortissement des infrastructures et des matériels et aux excédents de personnel attachés à ces médecins. L'impact de ces transferts médicaux est d'autant plus fort que certaines activités sont étroitement dépendantes de la présence de compétences médicales voisines : pas d'obstétrique sans chirurgie viscérale, pas de chirurgie vasculaire sans chirurgie cardiaque par exemple.

2. Le second obstacle à la réduction des coûts réside dans la difficulté de l'organisation hospitalière à adapter les nouveaux modes de production nécessaires pour développer les créneaux de développement identifiés. L'exemple le plus représentatif de cette inertie est la chirurgie ambulatoire, concentrée à plus de $70 \%$ dans le secteur privé lucratif ${ }^{17}$.

3. Le surdimensionnement hospitalier en termes d'immobilier comme de personnel constitue le troisième obstacle à la stratégie de réduction des coûts. Le différentiel de coût du mètre carré hospitalier public est ainsi estimé à près de $30 \%$ par rapport au coût de construction du privé. La productivité hospitalière des personnels est également plus faible. Cette sous-productivité est sans doute pour partie liée - mais pour partie seulement - aux contraintes de permanence des soins qui pèsent sur l'hôpital public, dont le surcoût n'a jamais été vraiment évalué. Cette obligation de permanence des soins rend difficile l'alignement des coûts de production avec les concurrents privés, eux aussi désormais financés par la T2A, et qui n'ont de cesse de demander une convergence tarifaire publique et privée.

La stratégie des hôpitaux publics pour faire face à la concurrence peut aussi reposer sur le développement des alliances. La notion de «stratégie de groupe publique » portée par la Fédération Hospitalière de France a ainsi conduit à la création dans la loi HPST de la communauté hospitalière de territoire (CHT), nouvel instrument présenté comme la clé de voûte de la recomposition du secteur hospitalier public.

Mesure phare du rapport Larcher d'avril 2008 sur les missions de l'hôpital, préconisant de s'inspirer du modèle des communautés de communes et d'agglomérations pour réaliser de grands ensembles publics à l'échelle d'un territoire, la CHT, réservée aux seuls établissements publics, vise à mettre en œuvre une stratégie commune et à gérer en commun certaines fonctions et activités grâce à des délégations ou des transferts de compétences entre les établissements et grâce à la télémédecine. Elle vise en fait essentiellement à préserver

\footnotetext{
${ }^{17}$ Calcul réalisé sur la base de données PMSI 2009.
} 
le maillage d'hôpitaux de proximité et d'hôpitaux locaux en créant des groupes publics territoriaux, à l'intérieur desquels pourrait être réorganisée l'offre dans le sens d'une plus grande efficience.

La communauté repose sur une convention passée entre les membres et approuvée par le directeur de l'ARS, qui définit notamment un projet médical commun, les compétences, activités et biens qui seront déléguées ou transférées entre les établissements partenaires, ainsi que les modalités de coopération entre les établissements en matière de gestion et les modalités de mise en commun des ressources humaines et des systèmes d'information.

En contraignant les membres à conjuguer à la fois leurs intentions stratégiques et les actions concrètes à mettre en œuvre pour concrétiser ces intentions, la CHT vise à contraindre les parties prenantes à dépasser le stade des seules déclarations de bonne intention qui ont caractérisé bon nombre d'opérations dites de recomposition hospitalière publique.

Les bénéfices théoriques de la CHT se heurtent cependant à des coûts de coordination élevés liés à la nécessiter de gérer des sites de production multiples. On peut par conséquent craindre que ces CHT, comme leurs ancêtres les syndicats interhospitaliers, démontrent un impact réel sur l'efficience de l'offre publique assez faible. Leur intérêt principal est de préparer la fusion de plusieurs établissements, comme l'ont été en leur temps les syndicats interhospitaliers, que la fusion soit délibérée, ou, cas le plus fréquent, imposée par l'ARS, puisque le directeur de cette dernière peut contraindre des établissements à passer une convention de $\mathrm{CHT}^{18}$, un GCS ou un GIP.

\section{Conclusion}

Le PMSI constitue un gisement majeur d'informations pour décrire et analyser la production hospitalière de soins : si l'utilisation de ces informations tend à se généraliser tant par les agences régionales de santé que par les établissements sanitaires, le système est loin d'avoir épuisé tout son potentiel informatif comme le montrent les travaux encore expérimentaux conduits sur l'analyse de la concurrence et des marchés au travers d'indicateurs tels que le HHI. La combinaison des données médico-économiques avec des données socio-démographiques et financières, et leur traitement au sein de systèmes d'information géographiques (SIG) ouvrent pour les années à venir des perspectives encore largement inexplorées dans la connaissance des «marchés » de la santé.

Les connaissances produites par ces informations s'avèrent paradoxalement de peu d'utilité pour les parties prenantes publiques, qu'il s'agisse des agences régionales de santé ou des hôpitaux, du fait de la difficulté pour ces dernières à mettre en œuvre les décisions découlant de leurs analyses : difficultés pour les agences à réguler l'offre sur un territoire face aux incitations concurrentielles induites par la T2A, difficultés pour les établissements publics à déployer leurs stratégies concurrentielles compte tenu des freins et des obstacles inhérents au recrutement et à la fidélisation de la ressource médicale, et de coûts de production peu flexibles.

Cette situation montre bien le paradoxe de la politique sanitaire française, qui combine un système de financement d'inspiration libérale encourageant la concurrence, et une démarche de régulation de l'offre visant à améliorer l'efficience de la dépense publique et à contrecarrer à cet effet les comportements concurrentiels des établissements (Pouvourville G., 2009) .

\footnotetext{
${ }^{18}$ Article L6131-2 du code de la santé publique
} 
Dans ce contexte, les cliniques privées apparaissent comme les principales bénéficiaires de cette transparence informationnelle. La plasticité que leur confère leur modèle organisationnel réticulaire (Piovesan D. et Claveranne J.-P., 2003) et leur statut de droit privé en termes de recrutement et de maîtrise des coûts leur permet de se saisir rapidement des opportunités identifiées grâce aux analyses médico-économiques pour adapter leur offre.

\section{Bibliographie}

AMAR A. et BERTHIER L., 2006, Le nouveau management public : avantages et limites, Services Governance and Public Policies : XVI International Reser Conférence, Lisbonne, september

ATIH, 2007, Guide de l'Étude Nationale de Coûts à méthodologie commune MCO (ENCC MCO), Lyon, 159 p.

AUBlET-CUVELIER B., (2003), Le Programme de Médicalisation des Systèmes d'Information, outil d'aménagement des territoires sanitaires, Thèse de Géographie physique, humaine, économique et régionale, Université Paul Valéry - Montpellier 3, Montpellier, 233 p.

BOULOCHER V., FLAMBARD S. et JEAN S. (2006), L'analyse d'un marché, Vuibert, Paris.

CAPGRAS J.-B. (2009), Le Programme de médicalisation des systèmes d'information (1982-2009): anamorphose et métamorphoses d'un dispositif de gestion, Thèse de Doctorat en Sciences de Gestion, Graphos, Université Jean Moulin Lyon 3, Lyon, 8 décembre 2009, 450 p.

CAPPS C.S., DRANOVE D., GREENSTEIN S., et al., 2002, Antitrust policy and hospital mergers : recommendations for a new approach, The Antitrust Bulletin, pp. 677-714

CARTER T., 1999, The aftermath of reengineering : downsizing and corporate performance, Haworth Press, New York,

CASH R., 2004, La tarification à l'activité : première année de mise en œuvre, Revue d'économie financière, $\mathrm{n}^{\circ} 76$, pp. 209-222

CLAVERANNE J.-P. et PASCAL C., 2004, Repenser les processus à l'hôpital : une méthode au service de la performance, Medica Editions, Paris,

CLAVERANNE J.-P. et PASCAL C., 2005, T2A et gestion hospitalière : la physiologie du changement, Gestion hospitalière, $\mathrm{n}^{\circ} 449$, pp. 609-613

CLAVERANNE J.-P., PASCAL C., CAPGRAS J.-B., et al., 2006, Evaluation des opérations de recomposition hospitalière, Rapport intermédiaire Phase 1, Graphos, Lyon, $14 \mathrm{p}$.

COCA E., 1998, L'ISA et le PMSI : Comment mieux gérer les établissements hospitaliers?, BERGERLEVRAULT,

COLDEFY M. et LUCAS-GABRIELLI V., 2008, Les territoires de santé : des approches régionales variées de ce nouvel espace de planification, IRDES, Document de travail, Paris, 28 p.

DE POUVOURVILLE G., JEUNEMAITRE A. et CONDAMIN F., 1988, L'hôpital en chantier : Les réformes hospitalières entre 1981 et $1986,157 \mathrm{p}$.

DELLINGER A. et BOLARD P., 2010, Étude de relations de mutation entre unités fonctionnelles d'un centre hospitalier par l'analyse structurale de réseaux, Pratiques et organisations de soins, vol. 41, n 4, pp. 341-348

DOBRUSZKES F., 2007, La quantification et la géographie de la concurrence aérienne : aspects méthodologiques et application à l'Europe, 05/2007

ENGEL F., KLETZ F., MOISDON J.-C., et al., 2000, La démarche gestionnaire à l'hôpital. Le PMSI., Seli Arslan,

FETTER R.B., AVERILL R., BRAND D., et al., 1991,DRGs : their design and development, Technical Report, Health Administration Press,

FETTER R.B. et FREEMAN J.L., 1986, Diagnosis Related Groups : Product Line Management within Hospitals, Academy of Management Review, vol. 11, n 1, pp. 41-54 
GABEL R.A., 1999, Operating room management, Butterworth-Heinemann, Boston,

GUILHOT N., 2008, L'hôpital de Beaujeu et son domaine : neuf siècles de combat pour le soin, Hôpital local de Beaujeu, Beaujeu,

HAAS S. et VIGNERON E., 2004, Territoires de santé : quelles méthodes ?, Gestion hospitalière, n 437, pp. $477-485$

HAAS S. et VIGNERON E., 2009, 10 ans de recompositions hospitalières en France, L. n. f. d. territoires, La Défense, $14 \mathrm{p}$.

HALL M. et TIDEMAN N., 1967, Measures of concentration, Journal of the American Statistical Association, vol. $62, \mathrm{n}^{\circ} 317$, pp. $162-168$

HATCHUEL A., MOISDON J.-C. et MOLET H., 1988, Budget global et groupes homogènes de malades, Politique et Management Public, vol.3, $\mathrm{n}^{\circ} 4$,

HERFINDAHL O.C., 1950, Concentration in the U.S. steel industry, Dissertation, Columbia University,

HIRSCHMAN A.O., 1945, National Power and the structure of foreign trade, University of California Press,

HIRTZLIN I., 2007, Economie de la santé, Editions Archétype 82, Paris,

KERN A. (2006), The use of key figures and its impact on activity, Thèse de sciences de gestion, ESSEC, Cergy, $288 \mathrm{p}$.

LEHTONEN T., 2007, DRG-based prospective pricing and case-mix accounting--Exploring the mechanisms of successful implementation, Management Accounting Research, vol. 18, n 3, pp. 367-395

LERNOUT T., LEBRUN L. et BRÉCHAT P.-H., 2007, Trois génération de schémas régionaux d'organisation sanitaire en quinze années : bilan et perspectives, Santé publique, ${ }^{\circ} 19, \mathrm{pp} .499-512$

LINDA R., 1976, Méthodologie de l'analyse de la concentration appliquée à l'étude des secteurs et des marchés, 26 p.

MILLER J.P., 1955, Measures of Monopoly Power and Concentration: Their Economic Significiance, in Business Concentration and Price Policy, N. B. o. E. Research, Princeton University Press, pp. 119-140

MILON A., 2008, Rapport d'information au nom de commission des Affaires sociales sur l'avenir de la chirurgie en France, $50 \mathrm{p}$.

MOISDON J.-C. et TONNEAU D., 1996, L'hôpital public et sa tutelle : la concurrence sous administration, Revue Française de Gestion, n 109 , pp. 80-91

NAEGELEN F. et MOUGEOT M., 1998, Asymétrie d'information et financement des hôpitaux. Une comparaison des modes d'organisation du système de santé, Revue économique, pp. 1323-1343

NOURY J.-F., 2000, La gestion médicalisée des établissements de santé : Le PMSI et l'information médicale, MASSON, MASSON, Paris,

OSBORNE D. et GAEBLER T., 1992, Reinventing government : how the entrepreneurial spirit is tansforming the public sector, Addison-Wesley Pub. Co., Reading, Mass.,

PASCAL C., CAPGRAS J.-B., BOTTON J.-F., et al., 2009, La mesure et l'analyse de la concurrence au service de la stratégie des établissements de santé, Journal d'Économie Médicale, vol. 27, n 1-2, pp. 21-42

PEREIRA C. et RAVISSOT S., 2007, Relations établissements/tutelle. Nouveaux enjeux et nouveaux outils de dialogue, Gestions hospitalières, $\mathrm{n}^{\circ}$ 463, pp. 135-141

PIOVESAN D. et CLAVERANNE J.-P., 2003, La clinique privée, un objet de gestion non identifié, Revue Française de Gestion, ${ }^{\circ}$ 146, pp. 143-153

POLLITT C., 1990, Managerialism and the public services : the Anglo-American experience, Basil Blackwell, Cambridge, Mass., USA,

POUVOURVILLE G., 2009, Les hôpitaux français face au paiement prospectif au cas : La mise en œuvre de la tarification à l'activité, Revue Economique, vol. 60, n² 2, pp. 457-470

RODRIGUES J.-M. et GOLDBERG S., 1984, Le Projet de Médicalisation des Systèmes d'Information (PMSI), Informations Hospitalières, vol. 1, pp. 15-19

SAVING T.R., 1970, Concentration ratios and the degree of monopoly, International Economic Review, vol. 11, $\mathrm{n}^{\circ} 1$, pp. 139-146 
SCHRAMM C.J. et RENN S.C., 1984, Hospitals mergers, market concentration and the Herfindahl-Hirschman Index, Emory Law Journal, n 33 , pp. 869-888

SCITOVSKY T., 1955, Economic theory and the measurement of concentration, in Business Concentration and Price Policy, N. B. o. E. Research, Princeton University Press, pp. 99-116

TITTON M., BINQUET C., VOURC'H M., et al., 2008, Suivi de l'activité cancérologique au CHU de Dijon : intérêt du PMSI, Santé publique, n 20, pp. 411-423

TOMASKO R.M., 1987, Downsizing : reshaping the corporation for the future, AMACOM, New York, 
Annexe

Liste des sigles utilisés dans I'article

\begin{tabular}{|c|c|c|}
\hline Sigles & Définitions & Explications \\
\hline PMSI & $\begin{array}{l}\text { Programme } \\
\text { de médicalisation } \\
\text { des systèmes d'information }\end{array}$ & $\begin{array}{l}\text { Mis en place sous la forme d'un projet au début des } \\
\text { années } 80 \text {, ce programme vise à décrire l'activité } \\
\text { hospitalière de court séjour à partir d'environ } 2300 \\
\text { groupes iso-ressources présentant également une } \\
\text { homogénéité médicale. }\end{array}$ \\
\hline GHM & $\begin{array}{l}\text { Groupe homogène } \\
\text { de malades }\end{array}$ & $\begin{array}{l}\text { Appellation donnée aux groupes iso-ressources } \\
\text { du PMSI. }\end{array}$ \\
\hline CMD & $\begin{array}{l}\text { Catégorie majeure } \\
\text { de diagnostic }\end{array}$ & $\begin{array}{l}\text { Les } 2368 \text { GHM du PMSI sont regroupés en } 28 \text { CMD } \\
\text { leur conférant leur homogénéité médicale. }\end{array}$ \\
\hline T2A & Tarification à l'activité & $\begin{array}{l}\text { A partir de 2004, un système de paiement prospectif } \\
\text { de l'activité de court séjour des établissements de } \\
\text { santé a été mis en place en France, fixant des tarifs } \\
\text { pour chaque GHM du PMSI. }\end{array}$ \\
\hline ENC & Etude nationale des coûts & $\begin{array}{l}\text { Mise en place par la circulaire DH/PMSI/92 n08 } \\
\text { du } 28 \text { février 1992, I'ENC vise à la constitution } \\
\text { d'une base de données sur les coûts des activités } \\
\text { hospitalières d'une sélection d'établissements } \\
\text { volontaires. Elle sert aujourd'hui de référentiel dans } \\
\text { la constitution de l'échelle tarifaire de la T2A. }\end{array}$ \\
\hline OQOS & $\begin{array}{l}\text { Objectif quantifié de l'offre } \\
\text { de soins }\end{array}$ & $\begin{array}{l}\text { Les OQOS fixent des seuils d'activité exprimés en } \\
\text { nombre d'implantations ou en volume de soins } \\
\text { pour chaque établissement de santé. }\end{array}$ \\
\hline SROS & $\begin{array}{l}\text { Schéma régional de } \\
\text { l'organisation sanitaire }\end{array}$ & 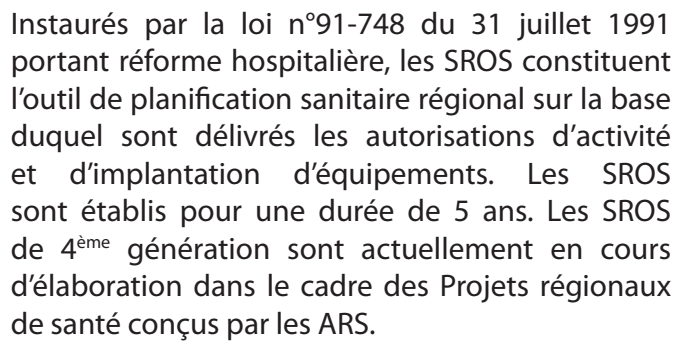 \\
\hline ONDAM & $\begin{array}{l}\text { Objectif national des } \\
\text { dépenses d'assurance } \\
\text { maladie }\end{array}$ & $\begin{array}{l}\text { Instauré par la loi organique du } 22 \text { juillet 1996, } \\
\text { I'ONDAM vise à encadrer les dépenses d'assurance } \\
\text { maladie par le vote annuel d'un objectif par le } \\
\text { parlement. }\end{array}$ \\
\hline FINESS & $\begin{array}{l}\text { Fichier national des } \\
\text { établissements sanitaires et } \\
\text { sociaux }\end{array}$ & $\begin{array}{l}\text { Ce répertoire national maintenu à jour par l'ARS } \\
\text { assure l'immatriculation des établissements et } \\
\text { entités juridiques porteurs d'une autorisation } \\
\text { ou d'un agrément sous la forme d'un identifiant } \\
\text { unique dit numéro FINESS. }\end{array}$ \\
\hline
\end{tabular}




\begin{tabular}{|c|c|c|}
\hline HHI & $\begin{array}{l}\text { Indice d'Herfindahl- } \\
\text { Hirschman }\end{array}$ & $\begin{array}{l}\text { Indice issu de l'économie industrielle mesurant la } \\
\text { concentration concurrentielle d'un marché. }\end{array}$ \\
\hline ARH & $\begin{array}{l}\text { Agence régionale } \\
\text { d'hospitalisation }\end{array}$ & $\begin{array}{l}\text { Créées par l'ordonnance n }{ }^{\circ} \text { 96-346 du } 24 \text { avril 1996, } \\
\text { les ARH sont des GIP associant les services de l'Etat } \\
\text { et de l'assurance maladie au niveau régional. Elles } \\
\text { planifiaient les structures et les équipements, et } \\
\text { allouaient les ressources aux établissements. }\end{array}$ \\
\hline ARS & $\begin{array}{l}\text { Agence régionale de la } \\
\text { santé }\end{array}$ & $\begin{array}{l}\text { Créées par la loi n } 2009-879 \text { du } 21 \text { juillet } 2009 \text { dite } \\
\text { loi « Hôpital, patients, santé et territoires », les ARS } \\
\text { regroupent et remplacent depuis le } 1^{\text {er }} \text { avril } 2010 \\
\text { les organismes jusque là en charge des politiques } \\
\text { de santé dans les régions et les départements. } \\
\text { Elles constituent désormais le pilote unique de la } \\
\text { régulation du système sanitaire au niveau régional. }\end{array}$ \\
\hline ATIH & $\begin{array}{l}\text { Agence technique } \\
\text { de l'information sur } \\
\text { I'hospitalisation }\end{array}$ & $\begin{array}{l}\text { Instituée par le décret n²000-1282 du } 26 \text { décembre } \\
2000 \text {, I'ATIH est chargée du pilotage et de la mise en } \\
\text { œuvre du PMSI et de la T2A. }\end{array}$ \\
\hline
\end{tabular}

${ }^{1}$ Le taux d'urbanisation de la zone de recrutement est calculé à partir de la part de population de la ZR résidant dans une commune qualifiée d'urbaine dans la typologie élaborée par I'INSEE. 